\title{
Effects of sex steroid hormone on regulation and localization of the calcium related genes in rat esophagus
}

\author{
Kipoong Kim, Song Ai Kang, Changhwan Ahn, Tran Dinh Nam and Eui-Bae Jeung \\ Laboratory of Veterinary Biochemistry and Molecular Biology, Chungbuk National University, \\ Cheongju, Chungbuk, 28644, Republic of Korea
}

\section{EP962: Steroid metabolism + action}

\section{INTRODUCTION}

Calcium ion is important for physiological functions in all tissues and organs, and is essential for many vital functions. Calcium in lumen is absorbed to cytosol, and Calbindin-D9k binds calcium. It can remove calcium to extracellular through PMCA1 and NCX1. However, in the rat esophagus, the calcium related gene regulations and localizations were not well documented. We evaluated calcium related genes including Calbindin-D9k, PMCA1, and NCX1.

\section{METHODS}

Immature rats were daily treated for three days with estradiol, progesterone and vehicle as control. To evaluate the pathway, antagonist groups were treated with $\mathrm{ICI} 182,780$ and RU486 before 30min ahead on hormone administration. The mRNA level of Calbindin-D9k, PMCA1, and NCX1 were quantified by qPCR, protein level and localization of Calbindin-D9k, PMCA1, and NCX1 in the esophagus were identified by immunofluorescence.

\section{RESULTS}

Figure 1. Transcriptional regulation of calcium related genes by steroid hormone in the esophagus.
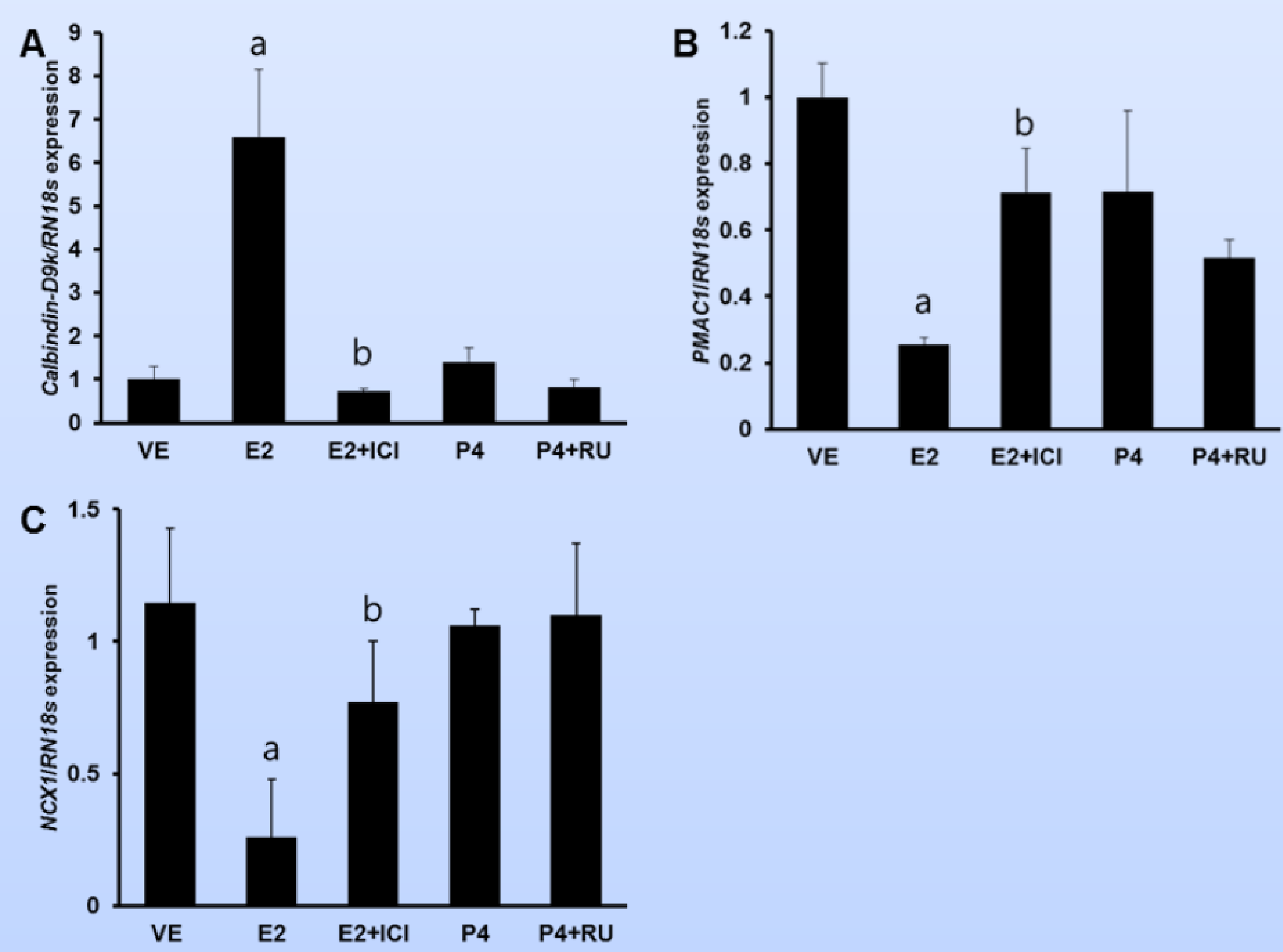

- The mRNA expression of Calbindin- $\mathrm{D}_{9 \mathrm{k}}(\mathrm{A}), \mathrm{PMCA} 1(\mathrm{~B})$, and NCX1 (C) in the esophagus was analyzed by real-time PCR. All genes expression was normalized to that of an internal control gene. Data are expressed as the mean $\pm \mathrm{SD}$ of at least three replicates. ${ }^{a} P<0.05$ versus vehicle, ${ }^{b} P<0.05$ versus agonists.

- Calbindin-D9k expression was increased by E2. PMCA1 expression was shown as opposed to the Calbindin-D9k expression. PMCA1 mRNA was decreased by E2. Similarly, NCX1 mRNA expression was decreased only by $E 2$.
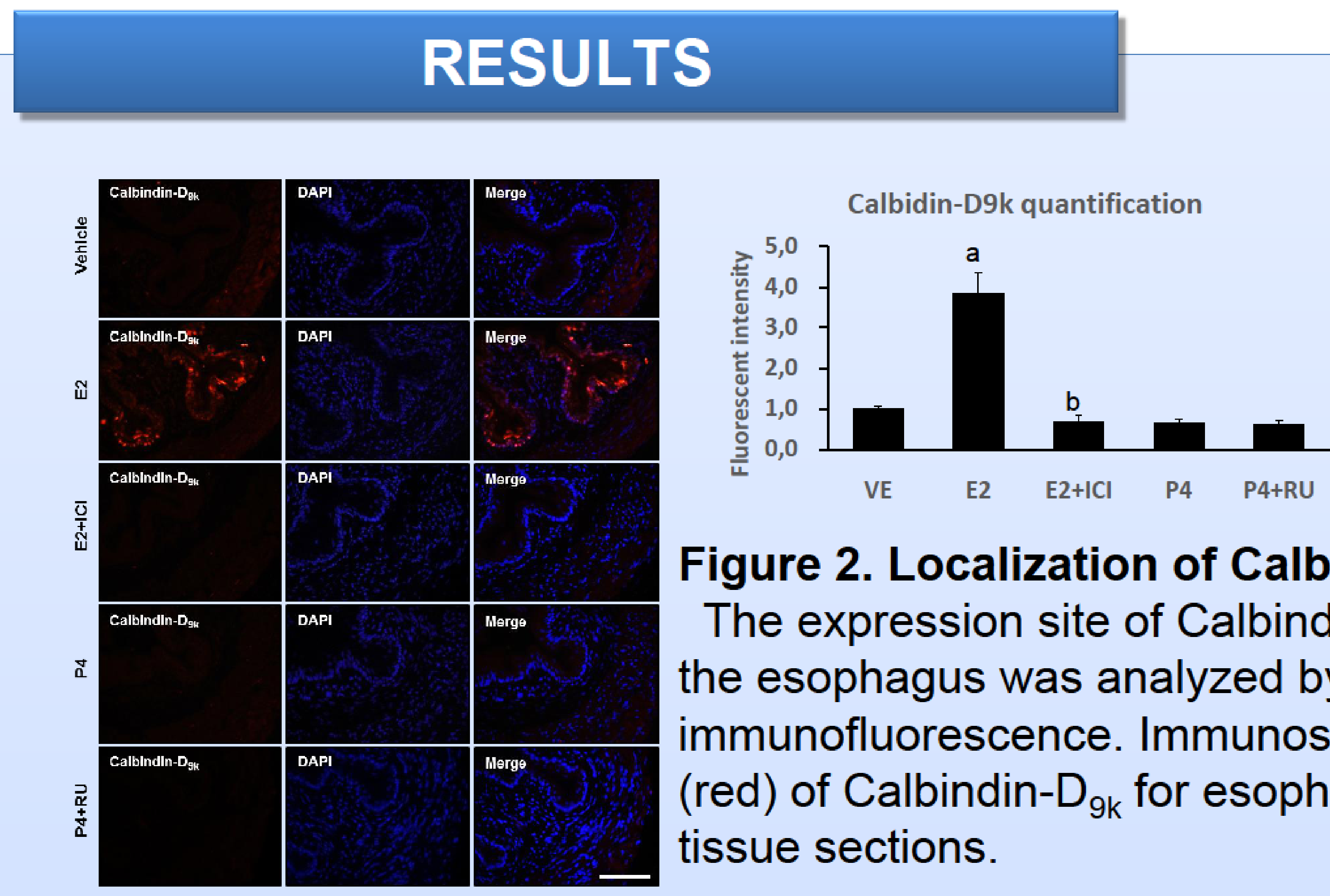

Figure 2. Localization of Calbindin- $\mathrm{D}_{9 \mathrm{k}}$ The expression site of Calbindin- $D_{9 k}$ in the esophagus was analyzed by immunofluorescence. Immunostaining (red) of Calbindin- $\mathrm{D}_{9 \mathrm{k}}$ for esophageal tissue sections.
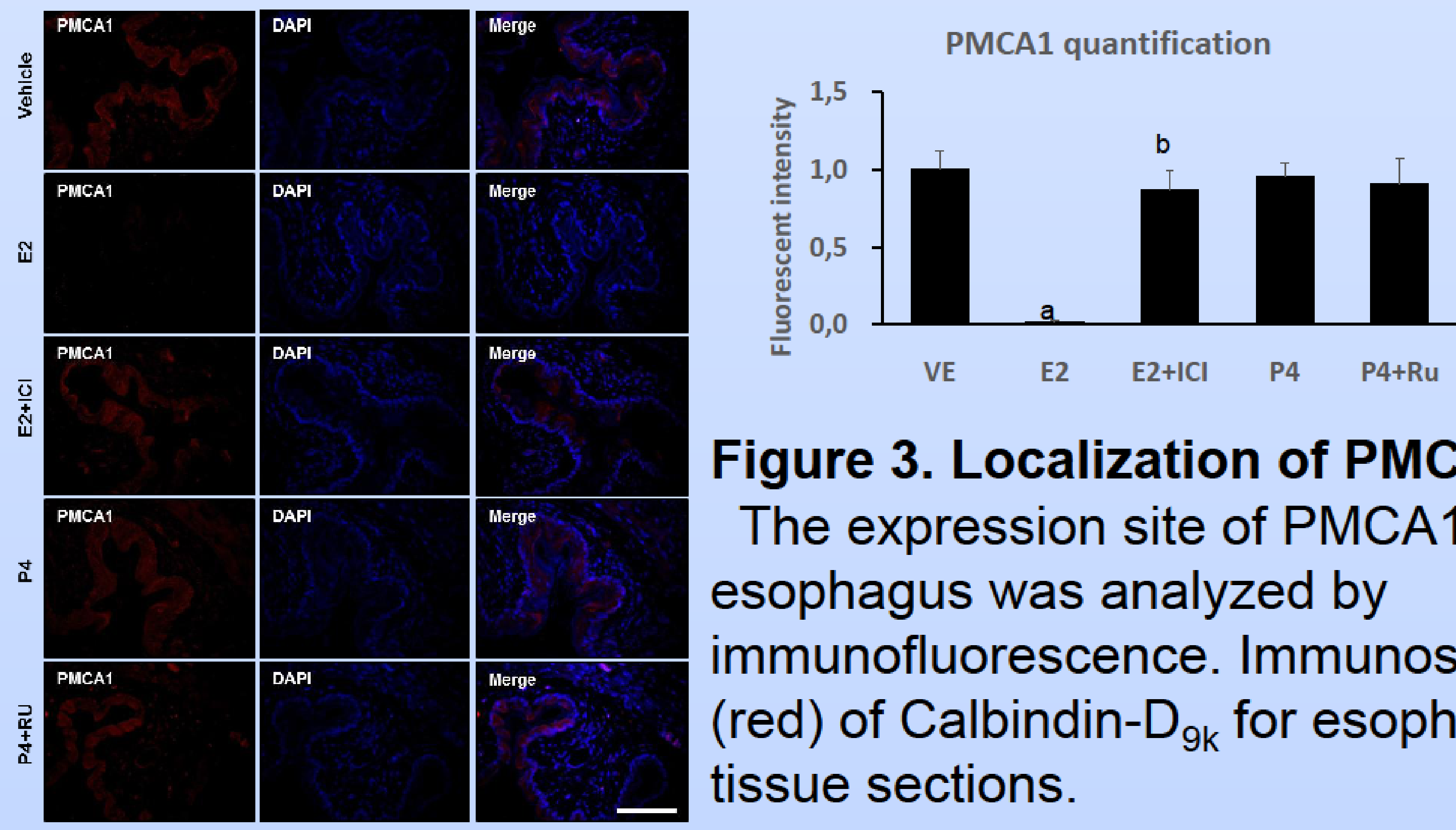

Figure 3. Localization of PMCA1

The expression site of PMCA1 in the esophagus was analyzed by immunofluorescence. Immunostaining (red) of Calbindin- $\mathrm{D}_{9 \mathrm{k}}$ for esophageal tissue sections.
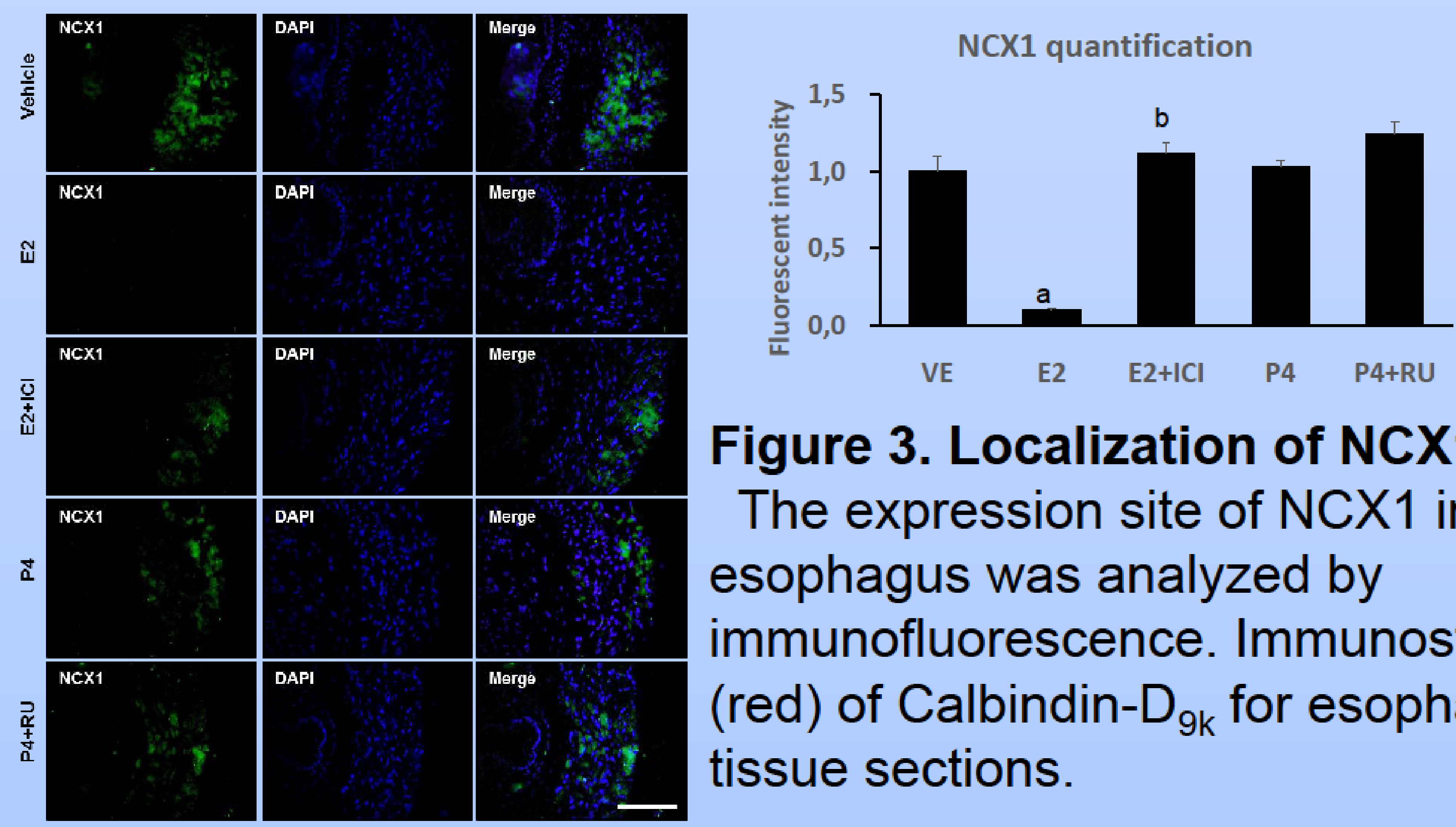

Figure 3. Localization of NCX1 The expression site of NCX1 in the esophagus was analyzed by immunofluorescence. Immunostaining (red) of Calbindin- $\mathrm{D}_{9 \mathrm{k}}$ for esophageal tissue sections.

- Scale bar represents $100 \mu \mathrm{m}$. Relative quantification of the immunostaining of Calbindin-D9k, PMCA1, and NCX1 is depicted in the bar graph by image $\mathrm{J}$. ${ }^{a} P<0.05$ versus vehicle, ${ }^{b} p<0.05$ versus agonists.

Calbindin-D9k and PMCA1 were mainly localized in mucosa layer. The main site of the NCX1 expression was muscularis externa.

\section{CONCLUSION}

Calcium related gene expressions were affected by steroid hormones in esophagus similar with previous studies. Study for regulation factor for Calcium related genes other than steroid hormone is further needed.

\section{REFERENCES}

Yang, H., An, B.S., Choi, K.C. and Jeung, E.B., 2013. Change of genes in calcium transport channels caused by hypoxic stress in the placenta, duodenum, and kidney of pregnant rats, Biol Reprod. $88,30$. 